\title{
Hanseníase e determinantes sociais em saúde no Sul do Brasil: Análise
}

\section{geograficamente ponderada}

Leprosy and Social Determinants of Health in South Brazil: Geographically evaluated analysis

Lepra y determinantes sociales de la salud en el sur de Brasil: Análisis ponderado geográficamente

Natalia Marciano de Araujo Ferreira

ORCID: https://orcid.org/0000-0002-5802-6188

Universidade Estadual de Londrina, Brasil

E-mail: natalia.marciano@outlook.com

Luiz Henrique Arroyo

ORCID: https://orcid.org/0000-0003-3302-0502

Escola de Enfermagem da Universidade de São Paulo, Brasil

E-mail: luiz.arroyo@hotmail.com

Thamy Barbara Gioia

ORCID: https://orcid.org/0000-0001-6431-6096 Universidade Federal de Goiás, Brasil

E-mail: thamygioia@gmail.com

Marcos Augusto Moraes Arcoverde

ORCID: https://orcid.org/0000-0001-5104-559X

Universidade Estadual do Oeste do Paraná, Brasil

E-mail: marcos.arcoverde2013@gmail.com

Ivaneliza Simionato de Assis

ORCID: https://orcid.org/0000-0003-1166-6644

Escola de Enfermagem da Universidade de São Paulo, Brasil E-mail: ivaneliza.assis@ @otmail.com

Marcelino Santos Neto

ORCID: https://orcid.org/0000-0002-6105-1886

Universidade Federal do Maranhão, Brasil

E-mail: marcelinosn@gmail.com

Mellina Yamamura

ORCID: https://orcid.org/0000-0001-5228-8788

Escola de Enfermagem da Universidade de São Paulo, Brasil

E-mail: mellinayamamura@yahoo.com.br

Alessandro Rolim Scholze

ORCID: https://orcid.org/0000-0003-4045-3584

Universidade Estadual do Norte do Paraná, Brasil

E-mail: scholze@uenp.edu.br

Ludmila Barbosa Bandeira Rodrigues

ORCID: https://orcid.org/0000-0001-5063-2039

Universidade Federal de Mato Grosso, Brasil

E-mail: ludbbremerick@gmail.com

Franciely Midori Bueno de Freitas

ORCID: https://orcid.org/0000-0003-0815-2765

Universidade Estadual de Londrina, Brasil

E-mail: franmidori@hotmail.com

Natacha Bolorino

ORCID: https://orcid.org/0000-0002-3039-2987

Universidade Estadual de Londrina, Brasil

E-mail: natachabolorino@ hotmail.com

Laís Cristina Gonçalves Ribeiro

ORCID: https://orcid.org/0000-0002-4522-3297

Universidade Estadual de Londrina, Brasil

E-mail: lcg.enf@ hotmail.com

Jessica Maia Storer

ORCID: https://orcid.org/0000-0002-9973-501X

Universidade Estadual de Londrina, Brasil

E-mail: jessicamaiast@gmail.com

Ricardo Alexandre Arcêncio

ORCID: https://orcid.org/0000-0003-4792-8714 


\title{
Flavia Meneguetti Pieri \\ ORCID: https://orcid.org/0000-0003-1239-2550 Universidade Estadual de Londrina, Brasil E-mail: fpieri@uel.br
}

\begin{abstract}
Resumo
Objetivo: verificar a relação entre hanseníase e espaço geográfico, levando em consideração determinantes sociais em saúde e utilizando ferramenta que permita a análise dessas relações localmente. Metodologia: Estudo ecológico, realizado em município endêmico do Sul do Brasil. Foi conduzida a geocodificação dos casos e selecionadas variáveis socioeconômicas de interesse. Após análise descritiva, seguiu-se com a correlação espacial por meio do Índice de Moran Bivariado, seguido de Regressão Linear. Para as variáveis presentes no modelo selecionado, aplicou-se a Geographically Weighted Regression por meio do software ArcGis versão 10.5.1. Na sequência, os resultados foram especializados por meio de mapas temáticos. Resultados: Em relação às características socioeconômicas e clinicooperacionais, houve maior quantidade de casos do sexo masculino (55,3\%), faixa etária de 16 a 60 anos (69,3\%), raça branca $(67,8 \%)$, casos multibacilares $(80,9 \%)$, forma clínica dimorfa $(45,2 \%)$ e grau de incapacidade I $(62,6 \%)$. A análise espacial demonstrou incidência média de 1,19 casos/10.000 habitantes, em cada setor censitário. Em relação aos determinantes sociais, as características se apresentam de forma heterogênea no espaço, sendo que aglomeração de habitantes apresentou associação direta com a hanseníase, bem como raça/cor parda, caracterizando-se como fatores de risco nas regiões de maior evidência. Em contrapartida, alfabetização apresentou relação inversa, associando o aumento dela com a redução na ocorrência da doença. Conclusão: Os determinantes sociais em saúde auxiliam na explicação da heterogeneidade da distribuição da doença. Compreender essa relação é fundamental para direcionar ações de profilaxia e controle.
\end{abstract}

Palavras-chave: Hanseníase; Saúde pública; Análise espacial; Atenção primária à saúde; Determinantes sociais da saúde.

\begin{abstract}
Objective: to verify the relationship between leprosy and geographic space, taking into account social determinants of health and using a tool that allows the analysis of these relationships locally. Methodology: Ecological study, carried out in an endemic city in southern Brazil. The geocoding of the cases was conducted and socioeconomic variables of interest were selected. After descriptive analysis, we followed with the spatial correlation through the Bivariate Moran Index, followed by Linear Regression. For the variables present in the selected model, the Geographically Weighted Regression was applied using the ArcGis software version 10.5.1. Subsequently, the results were specialized using thematic maps. Results: Regarding socioeconomic and clinical-operational characteristics, there was a greater number of male cases $(55.3 \%)$, aged 16 to 60 years $(69.3 \%)$, white race $(67.8 \%)$, multibacillary cases $(80.9 \%)$, borderline clinical form $(45.2 \%)$ and disability grade I $(62.6 \%)$. The spatial analysis showed an average incidence of 1.19 cases/10,000 inhabitants, in each census sector. Regarding social determinants, the characteristics are heterogeneous in space, and agglomeration of inhabitants showed a direct association with leprosy, as well as race/brown color, characterizing as risk factors in the most evident regions. On the other hand, literacy had an inverse relationship, associating an increase in literacy with a reduction in the occurrence of the disease. Conclusion: Social determinants in health help to explain the heterogeneity of disease distribution. Understanding this relationship is essential to direct prophylaxis and control actions.
\end{abstract}

Keywords: Leprosy; Public health; Spatial analysis; Primary health care; Social determinants of health.

\section{Resumen}

Objetivo: verificar la relación entre lepra y espacio geográfico, teniendo en cuenta determinantes sociales de salud y utilizando una herramienta que permita el análisis de estas relaciones a nivel local. Metodología: Estudio ecológico, realizado en ciudad endémica del sur de Brasil. Se realizó la geocodificación de casos y se seleccionaron variables socioeconómicas de interés. Luego del análisis descriptivo, se siguió con la correlación espacial a través del Índice Bivariante de Moran, seguido de Regresión Lineal. Para las variables presentes en el modelo seleccionado, se aplicó la regresión ponderada geográficamente utilizando el software ArcGis versión 10.5.1. Posteriormente, los resultados se especializaron mediante mapas temáticos. Resultados: En cuanto a las características socioeconómicas y clínicooperativas, hubo mayor número de casos masculinos $(55,3 \%)$, de 16 a 60 años $(69,3 \%)$, raza blanca $(67,8 \%)$, casos multibacilares $(80,9 \%)$, forma clínica límite. (45,2\%) y grado de discapacidad I (62,6\%). El análisis espacial mostró incidencia de 1,19 casos / 10.000 habitantes, en cada sector censal. Cuanto a los determinantes sociales, las características son heterogéneas en el espacio, y la aglomeración de habitantes mostró asociación directa con la lepra, así como la raza / color marrón, caracterizándo como factores de riesgo en las regiones más evidentes. Por otro lado, la alfabetización mostró una relación inversa, asociando un aumento de la alfabetización con una reducción en la ocurrencia de la enfermedad. Conclusión: Los determinantes sociales en salud ayudan a explicar la heterogeneidad de la distribución de las enfermedades. Comprender esta relación es fundamental para dirigir acciones de profilaxis y control. 
Palabras clave: Lepra; Salud pública; Análisis espacial; Atención primaria de salud; Determinantes sociales de la salud.

\section{Introdução}

A hanseníase é uma doença crônica e transmissível, cuja fragilidade não é relacionada diretamente a mortes, mas à incapacidade que causa aos indivíduos. Em um panorama global, no ano de 2014 a prevalência da doença foi de 0,31 casos por 10.000 habitantes, o que é aceito como positivo, visto que a meta de eliminação da Organização Mundial de Saúde (OMS) é uma prevalência menor de um caso para cada 10.000 habitantes. O Brasil, apesar de avanços significativos, é o segundo no ranking mundial e não atingiu essa meta, com a prevalência de 1,49 casos/10.000 habitantes, bem como o município em foco nesse estudo, com uma média aproximada de 1/10.000 hab. Por outro lado, o Paraná tem uma taxa menor, de 0,71/10.000 hab, ainda sendo a maior comparada com os outros estados da região Sul (OMS, 2016; Brasil, 2018).

Essa disparidade entre taxas de regiões diferentes salienta a importância de estudar o ambiente relacionando-o às doenças e chama a atenção para uma das subdivisões de pilares estratégicos da OMS em relação à hanseníase, que visa agregar a utilização de sistemas de informações geográficas com o objetivo de fortalecer sistemas de vigilância, informação, monitoramento e avaliação de programas (OMS, 2016). Paralelamente, em meados dos anos 2000, a mesma OMS criou a Comissão sobre Determinantes Sociais da Saúde (DSS) (Commission on Social Determinants of Health - CSDH), com o objetivo de promover, em âmbito internacional, uma tomada de consciência sobre a importância deles na situação de saúde de indivíduos e populações e sobre a necessidade do combate às iniquidades em saúde por eles geradas (CNDSS, 2008).

Os DSS, que são os fatores sociais, econômicos, culturais, étnico-raciais, psicológicos e comportamentais que são capazes de influenciar a ocorrência de problemas de saúde e de seus fatores de risco na população têm sido abordados e aceitos, direta ou indiretamente, como fatores medulares na investigação de doenças. De acordo com Marmot (2017), as iniquidades em saúde são inevitáveis, visto que decorrem de iniquidades na sociedade, presentes em todas elas. No âmbito das doenças infectocontagiosas e hanseníase especificamente, estudos conduzidos nacional e internacionalmente têm demonstrado a seriedade do local de habitação dos indivíduos, tanto na transmissão como na prevalência da doença, transformando o espaço em condição fundamental para estudos dos fatores de risco para a ocorrência dela (CSDH, 2008; Freitas, Duarte, \& Garcia, 2017; Silva et al., 2017; Brook et al., 2015).

A hanseníase é uma doença que pode formar aglomerados no espaço principalmente em áreas urbanas que possuem importantes índices de desigualdade social. Dessa forma, analisar a distribuição espacial da doença pode auxiliar na elaboração de hipóteses de fatores relacionados com ela. Além disso, é importante entender que analises espaciais que usam abordagens globais podem não discriminar heterogeneidades dentro de um mesmo cenário e, por isso, é oportuno o uso de fermentas estatísticas que analisem relações localmente como a Regressão Geograficamente Ponderada (Geographically Weighted Regression - GWR) (Ramos et al., 2017).

Revisão sistemática relacionando esses temas constatou a escassez de pesquisas conduzidas na região Sul do país, bem como estudos que analisam os determinantes sociais em saúde e a hanseníase de forma espacial com a utilização da ferramenta GWR. Sendo assim, o presente estudo apresenta a hipótese da existência de localidades com maior risco de adquirir a hanseníase na área em questão, bem como a relação dessa incidência com itens relacionados a determinantes sociais em saúde. Essa hipótese, caso confirmada, pode auxiliar nos programas do município visando o diagnóstico precoce e consequente melhoria na situação da doença no município. O objetivo desse estudo, então, foi verificar a relação existente entre hanseníase e espaço geográfico, levando em consideração determinantes sociais em saúde (quantidade de habitantes por domicílio, raça, escolaridade e renda) e utilizando ferramenta que permita a análise dessas relações localmente, em um município endêmico do Sul do Brasil. 


\section{Metodologia}

\section{Desenho do estudo}

Estudo ecológico (Morgenstern, 2008), ao qual foram utilizadas como unidade de análise os setores censitários e variáveis populacionais do município de Londrina-PR.

\section{População e fonte de dados}

O estudo consistiu de todos os casos de hanseníase diagnosticados e notificados do município de Londrina, localizado no norte do Paraná e a terceira cidade mais importante do sul do país com aproximadamente 558.439 habitantes (Londrina, 2017).

O município está organizado a partir das equipes de saúde da família, distribuídas nas 55 Unidades Básicas de Saúde (UBS), contando com 12 unidades em área rural e 43 em área urbana. Além de sete Núcleos de Apoio à Saúde da Família (NASF), conta ainda com 306 agentes comunitários de saúde (ACS) e 95 equipes de saúde da família (ESF), e com duas unidades de pronto atendimento (UPA). Ressalta-se que o atendimento a pacientes com hanseníase passou por descentralização a partir do ano de 2009 (Brasil, 2016b).

Os dados foram coletados no SINAN (Sistema de Informação de Agravos de Notificação), por meio das fichas eletrônicas de notificação de casos de hanseníase ocorridos entre os anos de 2009 e 2016. Foram registrados 426 novos casos de hanseníase nesse período e espaço. Dentre eles, 329 endereços residenciais foram geocodificados, constituindo a amostra do estudo. Os demais foram perdas (sete) ou não atenderam aos critérios de inclusão (53 eram residentes de outros municípios apesar do diagnóstico ter acontecido em Londrina e 37 residiam na zona rural).

\section{Geocodificação dos casos}

A geocodificação dos casos ocorreu por meio das informações de logradouro presentes nas fichas de notificação dos pacientes acometidos pela hanseníase. Foram utilizadas as coordenadas geográficas destes endereços residenciais (latitude e longitude), os quais foram obtidos por meio do software Google Earth® desenvolvido pela empresa Google. A geocodificação foi realizada utilizando-se o Software TerraView 4.2.2, que possibilitou elaborar um mapa de pontos dos pacientes com hanseníase nos setores censitários do município.

\section{Variáveis socioeconômicas}

Em relação aos determinantes sociais, foram utilizados dados do Censo 2010 do IBGE e escolhidos 13 (relacionados à transmissão da hanseníase), sendo estas variáveis apresentadas no Quadro 1. De acordo com o referencial teórico de Chaptini e Marshman (2015), foram agrupados em três dimensões:

a) Determinantes socioeconômicos: rendimento mensal per capita nos domicílios, pessoas alfabetizadas com cinco ou mais anos de idade e a proporção de pessoas de acordo com a raça/cor.

b) Determinantes ambientais: presença ou ausência de banheiro de uso exclusivo dos moradores em domicílios particulares permanentes e proporção de domicílios de acordo com o número de moradores. 
Quadro 1 - Determinantes Sociais em Saúde selecionadas para o estudo.

\begin{tabular}{|l|l|}
\hline Código & Variáveis \\
\hline V03 & Média do número de moradores em domicílios particulares permanentes \\
V04 & Domicílios particulares permanentes com banheiro de uso exclusivo dos moradores ou sanitário \\
V05 & Domić́lios particulares permanentes sem banheiro de uso exclusivo dos moradores e nem sanitário \\
V06 & Domić́lios Particulares Permanentes com até quatro moradores \\
V07 & Domicílios Particulares Permanentes com mais de cinco moradores \\
V08 & Pessoas alfabetizadas com cinco ou mais anos de idade \\
V09 & Pessoas residentes e cor ou raça branca \\
V10 & Pessoas residentes e cor ou raça preta \\
V11 & Pessoas residentes e cor ou raça amarela \\
V12 & Pessoas residentes e cor ou raça parda \\
V13 & Pessoas residentes e cor ou raça indígena \\
V14 & Domić́lios particulares com rendimento nominal mensal per capita de até um salário mínimo \\
V15 & Domicílios particulares com rendimento nominal mensal per capita de mais de um salário mínimo \\
\hline
\end{tabular}

Fonte: Autores (2021).

\section{Análise de dados}

Foi realizada análise descritiva dos casos, utilizando-se o programa IBM SPSS Statistics 20.0 (Statistical Package for the Social Sciences) para demonstrar as frequências relacionadas aos determinantes sociais em saúde e clínico operacionais encontradas nas fichas de notificação, sendo eles idade (variável contínua, categorizada em faixas etárias), raça, forma clínica, classificação operacional e GIF no diagnóstico.

O cálculo de incidência anual média entre os anos de estudo (2009-2016) padronizada foi realizado levando em consideração o sexo (masculino; feminino) e idade ( $\leq 14$ anos; 15 a 59 anos; $\geq 60$ anos) da população nos setores censitários de Londrina.

Para explicar a incidência dos casos nas unidades de análise do estudo, foram utilizadas as variáveis socioeconômicas citadas anteriormente. Foi conduzida uma correlação espacial por meio do Índice de Moran Bivariado. As variáveis que apresentaram correlação foram inseridas em uma Regressão Linear (OLS). O melhor modelo linear foi selecionado a partir dos valores de Akaike Information Criterion (AIC). Foi verificado a multicoliniaridade das variáveis através do Variance Inflation Factor (VIF), sendo considerado adequados valores inferiores a 10 (Montgomery, 2013). Além disso, o modelo foi analisado segundo estatística de Wald e de Koenker.

Para as variáveis que permaneceram no modelo OLS, aplicou-se a Geographically Weighted Regression (GWR) por meio do software ArcGis versão 10.5.1. O Índice de Moran (I) dos resíduos dos modelos resultantes do GWR foi avaliado quanto à dependência espacial. Mapas temáticos foram construídos no software ArcGis versão 10.5.1; para todos os testes, o erro tipo I foi fixado a $5 \%(\mathrm{p}<0,05)$ como estatisticamente significativo.

\section{Aspectos éticos}

Este estudo não possui fontes de financiamento e foi aprovado pelo Comitê de Ética em Pesquisa da UEL sob o parecer número CAAE 38642514.3.0000.5231. 


\section{Resultados}

As características socioeconômicas e clínico-operacionais dos indivíduos podem ser verificadas na Tabela 1 . Houve maior quantidade de casos do sexo masculino (182; 55,3\%), na faixa etária entre 16 a 60 anos com quase $70 \%$ do total registrado (228; 69,3\%). Em relação à raça, verificou-se maior quantidade de pacientes da raça branca, com 223 casos (67,8\%). Considerando as características específicas da doença, pode-se observar uma quantidade importante dos casos MB (80,9\%) e entre as formas clínicas a dimorfa (149 casos), 45,3\% do total, seguida da virchowiana (34,7\%). Ainda em relação à doença, mais da metade dos pacientes acompanhados já possuíam grau de incapacidade instalado no momento do diagnóstico (236; $71,7 \%)$.

Tabela 1 - Características sociodemográficas e clínico-operacionais dos casos de hanseníase, Londrina - PR (2009 - 2016).

\begin{tabular}{|c|c|c|}
\hline Variáveis & $\mathrm{N}=329$ & $\%$ \\
\hline \multicolumn{3}{|l|}{ Sexo } \\
\hline Masculino & 182 & 55,3 \\
\hline Feminino & 147 & 44,7 \\
\hline \multicolumn{3}{|l|}{ Faixa Etária } \\
\hline$<15$ anos & 8 & 2,4 \\
\hline 16 a 59 anos & 228 & 69,3 \\
\hline$>60$ anos & 93 & 28,3 \\
\hline \multicolumn{3}{|l|}{ Raça } \\
\hline Branco & 223 & 67,8 \\
\hline Negro & 29 & 8,8 \\
\hline Amarelo & 4 & 1,2 \\
\hline Pardo & 59 & 17,9 \\
\hline Indígena & 2 & 0,6 \\
\hline Não respondido & 12 & 3,7 \\
\hline \multicolumn{3}{|l|}{ Forma Clínica } \\
\hline Indeterminada & 22 & 6,7 \\
\hline Tuberculóide & 44 & 13,4 \\
\hline Dimorfa & 149 & 45,2 \\
\hline Virchowiana & 114 & 34,7 \\
\hline \multicolumn{3}{|c|}{ Classificação Operacional } \\
\hline Paucibacilar & 63 & 19,1 \\
\hline Multibacilar & 266 & 80,9 \\
\hline \multicolumn{3}{|c|}{ Grau de Incapacidade no Diagnóstico } \\
\hline Grau 0 & 82 & 24,9 \\
\hline Grau 1 & 206 & 62,6 \\
\hline Grau 2 & 30 & 9,2 \\
\hline Não avaliado & 11 & 3,3 \\
\hline
\end{tabular}

Fonte: Autores (2021). 
A partir da localização dos casos nos setores censitários urbanos do município, foram calculadas as taxas de incidência padronizadas por sexo e idade da doença (Figura 1). A incidência média nos anos de estudo foi de 1,19 casos por 10 mil habitantes, sendo que mais de $31 \%$ dos setores possuíam pelo menos um caso. Foi possível verificar uma distribuição dos casos por praticamente todo o espaço urbano do município, mesmo havendo uma grande quantidade de áreas com ausência de casos. Todavia, o município apresentou uma disseminação importante da hanseníase por áreas que provavelmente apresentam características distintas entre si.

Figura 1 - Taxa de incidência anual média de hanseníase (casos/10 mil habitantes) por setores censitários de Londrina, PR (2009-2016).

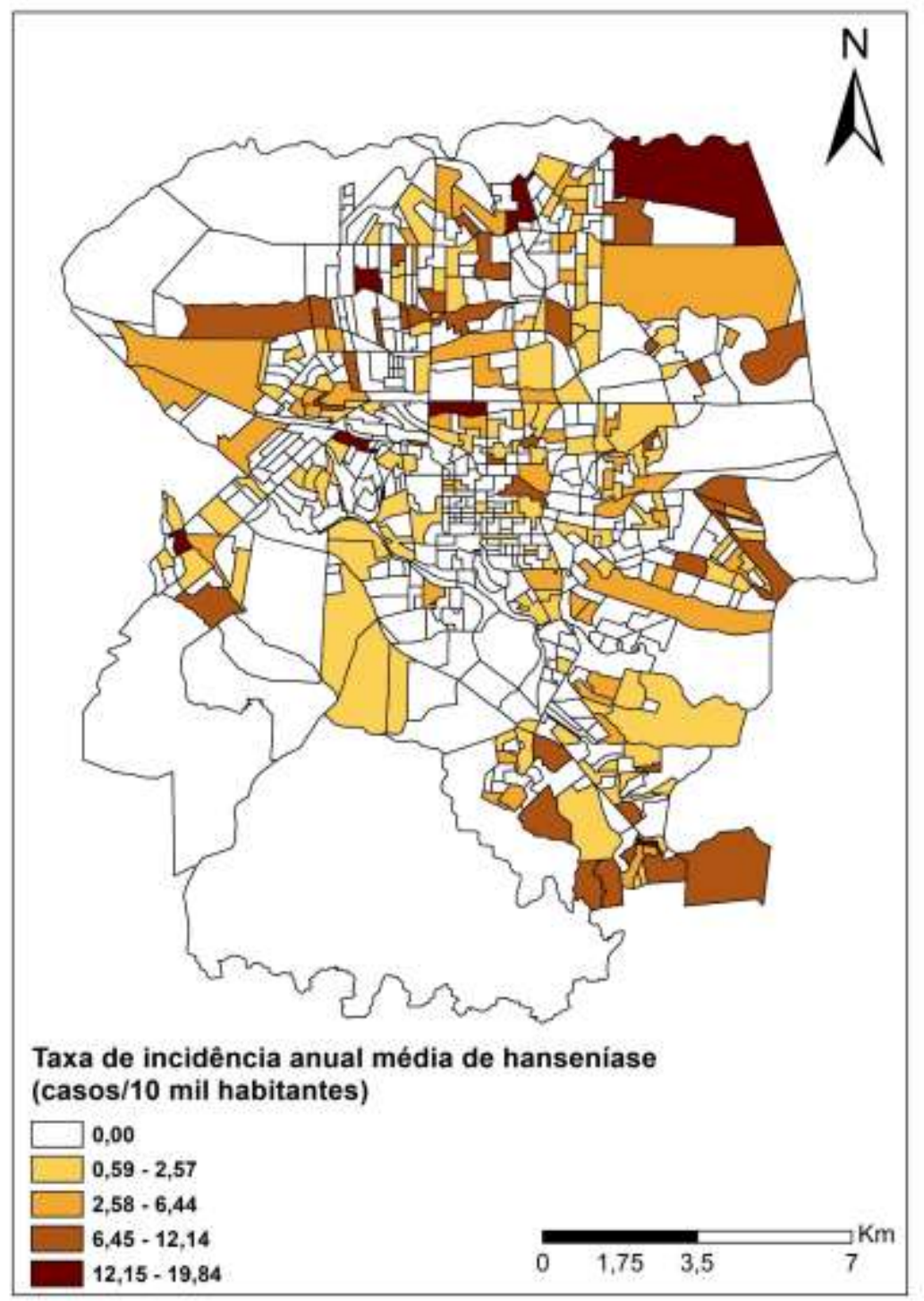

Fonte: Autores (2021).

Essa heterogeneidade pode ser visualizada na cidade como um todo, onde mais de 28.000 pessoas sobrevivem com menos de 1/4 de salário mínimo, distribuídas predominantemente na área urbana (95\%). Ao mesmo tempo, quanto mais dirigese às periferias, aumenta o percentual dos que ganham menos. Por exemplo, os bairros centrais são aqueles que concentram a maior parte das pessoas com rendimento superior a 20 salários mínimos, enquanto na periferia sul 61 a $85 \%$ da população apresenta um rendimento de até 3 salários mínimos. Na periferia está também concentrada uma maior quantidade de 
domicílios mais populosos (Barros et al., 2008).

Em relação ao analfabetismo, segue-se a mesma tendência. Há uma maior quantidade de escolas estaduais no centro e bairros adjacentes, com taxa de analfabetismo de 7,4\%. Já nas periferias, essa taxa pode atingir entre 10 e $29 \%$ da população. As mais de 50 ocupações também constituem importante fator a ser observado, visto que abrigam aproximadamente 50.000 habitantes, distribuídos em todo o espaço, com ênfase na região sudeste (Barros et al., 2008).

Dessa forma, para compreender os determinantes sociais em saúde associados à ocorrência da doença no espaço, foram realizadas as correlações espaciais destas variáveis com as taxas de incidência por meio do Índice de Moran Bivariado (Tabela 2). Das 13 variáveis selecionadas, 12 apresentaram relação com a ocorrência da doença, a única que não apresentou correlação espacial foi a proporção de residentes com cor ou raça indígena.

Tabela 2 - Resultados Índice de Moran Bivariado e Regressão linear da taxa de incidência de hanseníase com determinantes sociais em saúde.

\begin{tabular}{|c|c|c|c|c|}
\hline & & Moran I & Regressão linear & \\
\hline Código & Variáveis & $\begin{array}{l}\text { Bivariado } \\
\text { (valor p) }\end{array}$ & $\begin{array}{l}\text { Coeficientes } \\
\text { robustos (valor p) }\end{array}$ & VIF \\
\hline V03 & $\begin{array}{l}\text { Média do número de moradores em domicílios particulares } \\
\text { permanentes }\end{array}$ & $0,131(>0,01)^{*}$ & $0.280(0.03)$ & 1,32 \\
\hline V04 & $\begin{array}{l}\text { Domicílios particulares permanentes com banheiro de uso } \\
\text { exclusivo dos moradores ou sanitário }\end{array}$ & $-0,052(>0,01)^{*}$ & - & \\
\hline V05 & $\begin{array}{l}\text { Domicílios particulares permanentes sem banheiro de uso } \\
\text { exclusivo dos moradores e nem sanitário }\end{array}$ & $0,052(>0,01)^{*}$ & - & \\
\hline V06 & Domicílios Particulares Permanentes com até quatro moradores & $-0,065(>0,01)^{*}$ & - & \\
\hline V07 & $\begin{array}{l}\text { Domicílios Particulares Permanentes com mais de cinco } \\
\text { moradores }\end{array}$ & $0,058(>0,01)^{*}$ & $0.011(0.02)$ & 1,62 \\
\hline V08 & Pessoas alfabetizadas com cinco ou mais anos de idade & $-0,033(\mathrm{p}=0,01)^{*}$ & $-0.001(0.02)$ & 1,92 \\
\hline V09 & Pessoas residentes e cor ou raça branca & $-0,075(>0,01)^{*}$ & - & \\
\hline V10 & Pessoas residentes e cor ou raça preta & $0,101(>0,01)^{*}$ & - & \\
\hline V11 & Pessoas residentes e cor ou raça amarela & $-0,140(>0,01)^{*}$ & - & \\
\hline V12 & Pessoas residentes e cor ou raça parda & $0,123(>0,01)^{*}$ & $0.002(0.01)$ & 2,33 \\
\hline V13 & Pessoas residentes e cor ou raça indígena & $-0,002(0,46)$ & - & \\
\hline V14 & $\begin{array}{l}\text { Domicílios particulares com rendimento nominal mensal per } \\
\text { capita de até um salário mínimo }\end{array}$ & $0,096(>0,01)^{*}$ & - & \\
\hline V15 & $\begin{array}{l}\text { Domicílios particulares com rendimento nominal mensal per } \\
\text { capita de mais de um salário mínimo }\end{array}$ & $-0,141(>0,01)^{*}$ & $-0.001(0.07)$ & 1,24 \\
\hline
\end{tabular}

* Probabilidade para erro tipo I menor que 5\% - valores estatisticamente significativos $\mathrm{R}^{2}=0,057 ; \mathrm{R}^{2}$ ajustado=0,049; AIC $=2990.86$; Wald= $79,04(<0,01)$. Fonte: Autores (2021).

As variáveis que apresentaram correlação espacial foram inseridas em um modelo de Regressão linear (OLS) (Tabela 5). O melhor modelo foi selecionado por meio do valor AIC, no qual quatro variáveis tiveram associação com a doença nesta análise multivariada e uma se manteve no modelo final devido ao seu papel de regulação. A média do número de moradores 
por domicílio $(\beta=0,280)$, domicílios com mais de cinco moradores $(\beta=0,011)$ e a proporção de residentes com cor/raça parda nos setores censitários $(\beta=0,002)$, tiveram relação direta com a incidência da hanseníase no município. Isto evidencia que a aglomeração de indivíduos em um mesmo domicílio e regiões com maior razão de pardos perante outras características de raça/cor, relacionam-se com maiores taxas de incidência. A proporção de pessoas alfabetizadas apresentou relação inversa com a incidência $(\beta=-0,001)$, assinalando que um aumento na alfabetização dos habitantes da zona urbana do município reduziria a ocorrência da hanseníase. O rendimento mensal per capita de mais de um salário mínimo apresentou uma relação igualmente inversa, todavia, não apresentou evidência de associação com a hanseníase $(\mathrm{p}=0,07)$. Entretanto, devido ao seu importante papel de regulação das outras variáveis e por ser considerada um importante determinante social em saúde, o mesmo foi mantido no modelo final.

Não foi verificada efeito de colinearidade entre as variáveis explicativas (valor VIF das variáveis menor que 7,5), o modelo OLS final pode explicar 5,7\% (Coeficiente de determinação - $\mathrm{R}^{2}$ ) da variação das taxas incidência e o valor de AIC foi de 2990,86. A estatística de Wald significativa confirma a associações das variáveis explicativas com a incidência.

Dessa forma, com as mesmas variáveis do modelo OLS, foi realizado o GWR (Figura 2), ao qual apresentou resultados melhores de coeficiente de determinação $\left(R^{2}=0,135\right)$, coeficiente de determinação ajustado $\left(R^{2}\right.$ ajustado=0,089) e AIC (2974,70). Assim, foi possível aumentar a capacidade de explicação da variação da variável resposta ao introduzir os coeficientes locais (análise espacial local). Os resíduos do modelo GWR não apresentaram autocorrelação espacial (Índice de Moran $=-0,003 ; \mathrm{p}=0,76$ ), significando uma aleatoriedade no espaço desses resíduos e assegurando a fidedignidade dos resultados do modelo espacial local. Além disso, verificou-se que os coeficientes de determinações locais ( $\mathrm{R}^{2}$ local), explicaram até $24 \%$ da variação da incidência em setores censitários ao sul do município (Figura 3). 
Figura 2 - Coeficientes locais dos DSS da Regressão Geograficamente Ponderada dos casos de hanseníase em Londrina, PR (2009-2016).

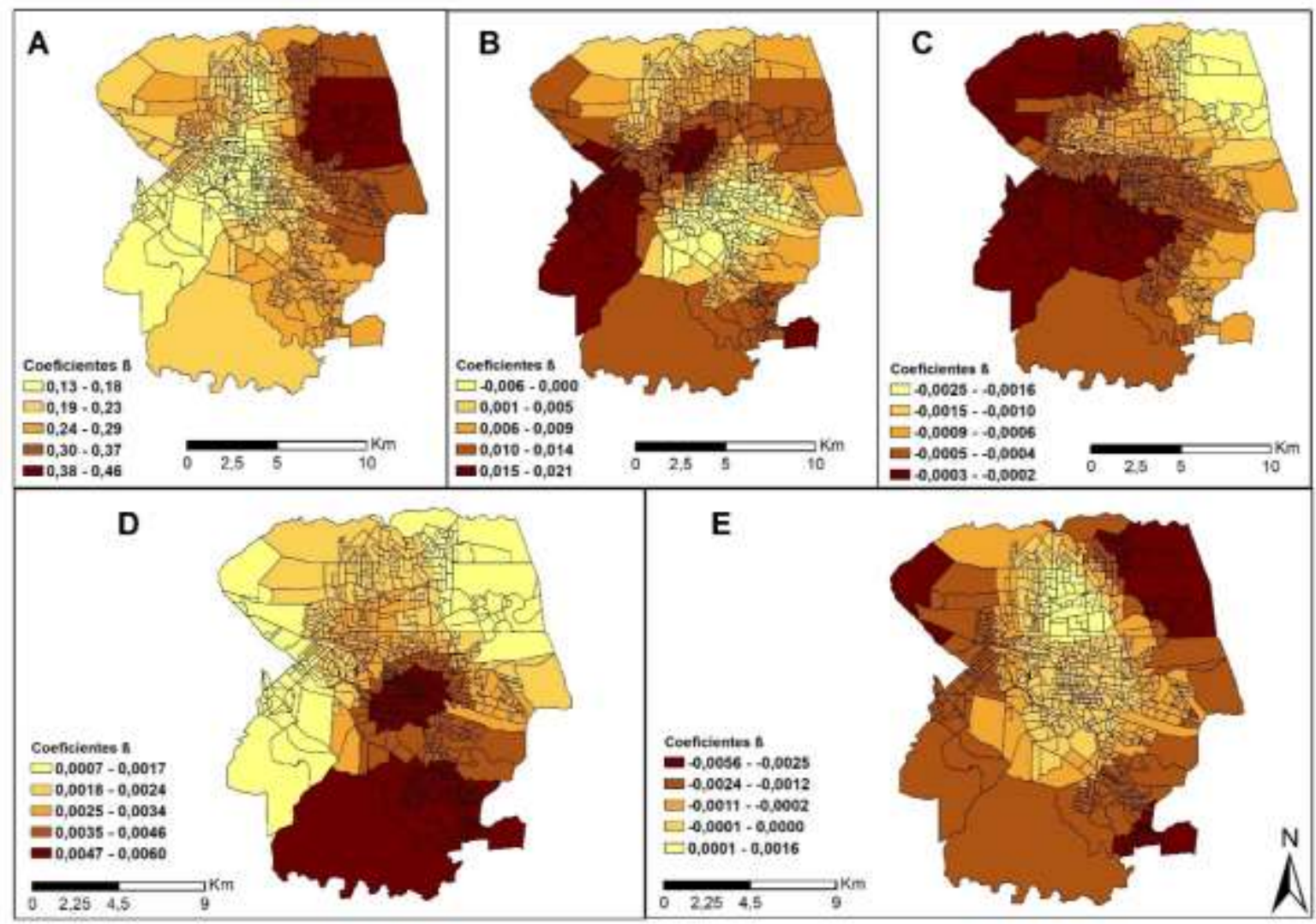

Legenda A: Média do número de moradores em domicílios particulares permanentes; B: Domicílios Particulares Permanentes com mais de 5 moradores; C: Pessoas alfabetizadas com 5 ou mais anos de idade; D: Pessoas residentes e cor ou raça parda; E: Domicílios particulares com rendimento nominal mensal per capita de mais de 1 salário mínimo. Fonte: Autores (2018). 
Figura 3 - Coeficientes de determinação local ( $\mathrm{R}^{2}$ local) da Regressão Geograficamente Ponderada dos casos de hanseníase em Londrina, PR (2009-2016).

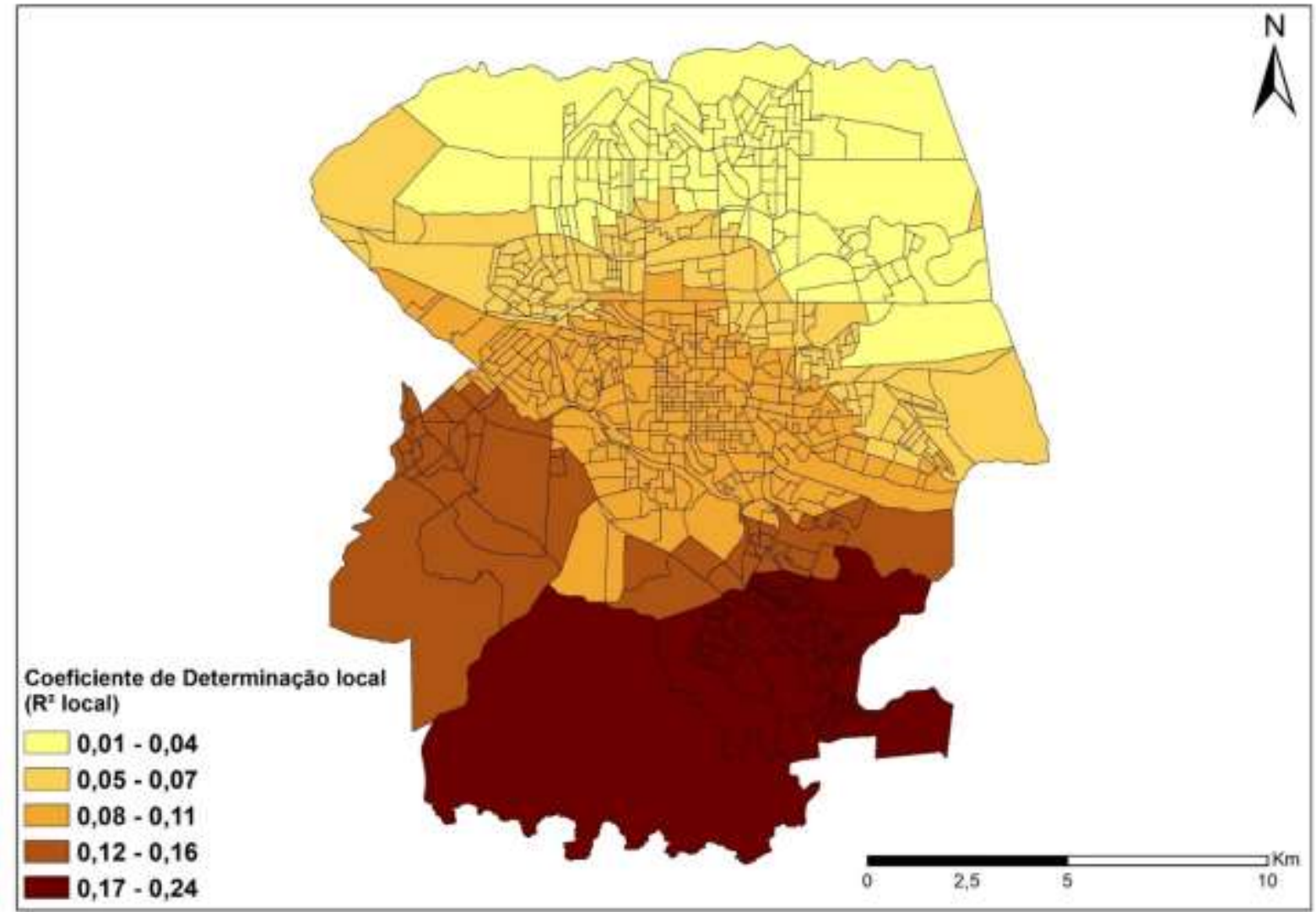

Fonte: Autores (2021).

As variáveis que representaram a aglomeração de habitantes, sendo estas a proporção de moradores por domicílio e a proporção de domicílios com mais de cinco moradores, apresentaram associações direta com a hanseníase, exibindo distinções pelas áreas urbanas de Londrina, mesmo ambas tendo similaridades em sua função de descrever a densidade domiciliar. Foi observado que na região nordeste do município o aumento do número de moradores teve forte relação com a hanseníase, independentemente da quantidade de indivíduos existentes nos domicílios. Em contraponto, na região sudoeste e sul essa relação foi mais forte apenas para o aumento de domicílios com mais de cinco moradores nos setores censitários.

O número de pessoas alfabetizadas nas unidades de análise foi um determinante com maior associação com a hanseníase a noroeste, oeste, sudoeste e sul de Londrina, sendo que esta apresentou relação inversa com o aumento da taxa de incidência em todo o cenário estudado. A razão de indivíduos que se classificaram como de raça/cor parda teve predominantemente relação positiva, destacando maior impacto em regiões centrais, sudeste e sul. Por fim, mesmo não tendo evidência de associação, o rendimento mensal de mais de um salário mostrou seu papel de regular o modelo principalmente por estar com maior relação linear com a incidência da hanseníase nas áreas periféricas de Londrina, locais nos quais houve maiores coeficientes de determinação local ( $\mathrm{R}^{2}$ local) das análises do GWR.

\section{Discussão}

Foi verificada importante relação entre hanseníase, local de moradia do paciente e DSS (quantidade de habitantes por domicílio, raça, escolaridade e renda), com uma evidente heterogeneidade desta associação no espaço urbano do município. A análise espacial tem sido conduzida em outros cenários do Brasil e tem demonstrado semelhantes características, apresentando 
aglomerados de ocorrência da doença e relação da incidência com as condições de vida da população (Silva et al, 2017. Freitas et al., 2017). Entre os países que apresentam também essa heterogeneidade, pode-se destacar a Índia, como aborda Brook et al. (2015) em seus achados.

Em relação ao perfil dos casos de hanseníase, verificou-se uma similaridade deste com outro estudo da literatura. O sexo masculino frequentemente encontra-se em evidência quando o tema é a doença em questão. Estudo conduzido por Ramos et al. (2017) verificou essa característica, inclusive em relação à multibacilaridade. Como nessa pesquisa, os diagnósticos encontrados já apresentavam classificação multibacilar e grau I de incapacidade já no momento do diagnóstico.

A OMS tem abordado o tema dos DSS com muita seriedade. No relatório da comissão responsável, em 2008, ela traz esses determinantes como sendo um tópico que necessita de uma visão holística. Nesse documento, explica que há uma divisão desigual de experiências que possam causar danos à saúde, e a combinação de fatores que a determinam têm grande responsabilidade por grande parte das fragilidades relacionadas à saúde (CSDH, 2008).

Trazendo a hanseníase para um plano de evidência, outros autores encontraram a relação dela com esses e outros determinantes. Freitas et al. (2014) encontrou que a maior incidência de casos em seu local de estudo foi registrada em municípios com maior população, maior taxa de pobreza e desemprego. Cunha et al. (2016), por sua vez, verificou relação da incidência com o número de casas que possuíam água encanada. Com vistas a não apenas comprovar a relação dos determinantes como também demonstrar que quando esses indicadores melhoram há uma consequência direta em doenças como a hanseníase, Nery (2014) apresentou que programas governamentais de distribuição de renda têm influência direta com a melhoria do panorama da hanseníase, visto que melhoram a qualidade de vida da população.

Ainda no estudo de Freitas et al. (2014), foi verificada uma relação direta entre o número de moradores por domicílio e a incidência. Neste estudo, foi identificada uma situação similar. Como apresentado no mapa, é possível inferir que algumas áreas de Londrina, em especial ao sul, sudoeste e nordeste do município, apresentam uma forte relação com a incidência da enfermidade. Faz-se necessário, então, estreitar os programas existentes nessas regiões. Como exemplo de ações, pode-se citar a busca de contatos intradomiciliares, visto que apenas $15 \%$ do total de diagnosticados nesse período tiveram como modo de detecção o exame de contatos. Situação diferente foi encontrada em outras localidades como Salvador, onde mais de 50\% dos pacientes estudados reportaram ter contato com outro indivíduo infectado dentro do ambiente domiciliar, e cidades do Pará, onde a probabilidade encontrada de desenvolver hanseníase quando em contato intradomiciliar com doentes chegou a $85 \%$ (Freitas et al., 2014; Santos, 2016; Barreto, 2015).

A questão relacionada à etnia traz um contraponto interessante, visto que em um panorama geral há uma grande quantidade de indivíduos brancos portadores da doença, quantidade maior que todas as outras etnias agrupadas. Entretanto, quando verificada a relação com a incidência, o modelo que melhor explicou essa relação mostrou a etnia parda como fator de risco para adquirir a mesma, em setores ao sul, oeste e noroeste do município.

O nível de alfabetização da população apresentou uma importante associação com a hanseníase, demonstrando como são necessárias intervenções voltadas à educação em saúde. Estudo do ano de 2014 traz essa relação de forma muito forte, avaliando os pacientes que apresentavam falhas no tratamento como de escolaridade baixa, além da renda (Lopes, 2014).

A renda é considerada um importante DSS. Sua associação com os níveis de saúde da população já fora evidenciada por diversos outros autores (Nery, 2014; Silva et al., 2016). No presente estudo, a hanseníase apresentou tal associação sem significância estatística, todavia, por intermédio desta variável foi possível encontrar o melhor modelo explicativo para a doença no município. Considera-se que a permanência da renda tem um cunho regulador para explicar a hanseníase, pois a maioria das variáveis do estudo estão de alguma forma interligadas à renda, isto se sobressai nas análises independente de níveis de significância. De alguma forma, que não foi possível ser explicado nas análises realizadas, a renda interferiu na 
incidência da doença, sendo que isto pode ser verificado em estudos futuros que se utilizem de outras abordagens metodológicas.

Como limitações, o estudo apresenta a utilização de dados secundários obtidos do SINAN. Devido ao não preenchimento integral da ficha de notificação, pode haver incompletude dos dados. Além disso, áreas identificadas como de proteção podem ser na verdade áreas de subnotificação, provocando interpretação equivocada dos resultados. Outra limitação foi a dificuldade em encontrar estudos que utilizassem essa metodologia específica (GWR) visando comparação.

O estudo avançou no conhecimento da hanseníase na região Sul do país e em sua relação com alguns DSS. A principal evidência identificada foi a associação da doença com fatores sociais, como número de moradores por domicílio, alfabetização, etnia e renda.

\section{Conclusão}

Promover a simples distribuição dos casos da doença no espaço permite aos serviços de saúde focar suas ações em regiões com maior incidência. Conhecer quais são esses DSS permite o planejamento de ações voltadas não somente ao controle da doença, como também profiláticas, visto que são desvelados os nós críticos que podem levar a seu acontecimento, a fim de evitá-los, ainda que conhecê-los meramente não é suficiente almejando a eliminação da hanseníase, como ressaltado por Assis et al (2018) em estudo conduzido também no sul do Brasil. É necessário que haja avanços sociais a fim de alcançar essa eliminação.

A análise espacial por meio do GWR foi essencial para discriminar as heterogeneidades existentes dentro do município. Dessa forma, essa é uma importante ferramenta para ser considerada em estudos futuros na confirmação das evidencias encontradas no presente estudo.

\section{Referências}

Assis, I. S., Arcoverde, M. A. M., Ramos, A. C. V., Alves, L. S., Berra, T. Z., Arroyo, L. H. et al. (2018). Social determinants, their relationship with leprosy risk and temporal trends in a triborder region in Latin America. PLoS Negl Trop Dis, 14 (4).

Barreto, J. G., Bisanzio, D., Frade, M. A. C., Moraes, T. M. P, Gobbo, A. R., Guimarães, L. S., Silva, M. B., Prokopec, G. M. V., Spencer, J. S., Kitron, U., Salgado, C. G. (2015). Spatial epidemiology and serologic cohorts increase the early detection of leprosy. BMC Infectious Diseases, (15).

Barros, M. V. F., Archela, R. S., Barros, O. N. F., Gratão, L. H., Thery, H., Mello, N. A. (2008). Atlas Ambiental da Cidade de Londrina. http://www.uel.br/revistas/atlasambiental/

Braker, W. H., Sihombing, B. Djarir, H., Beise, K., Kusumawardhani, L., Yulihane, R. et al. (2012). Disability in people affected by leprosy: the role of impairment, activity, social participation, stigma and discrimination. Global Health Action (5).

Brasil. Ministério da Saúde. Sala de Apoio à Gestão Estratégica (SAGE). (2016). Relatório Gerencial. http://sage.saude.gov.br/?link=sistemas/relatorio

Brasil. Ministério da Saúde. DATASUS, Portal da Saúde. http://tabnet.datasus. gov.br

Brasil. Ministério da Saúde. Secretaria de Vigilância em Saúde. (2018). Boletim Epidemiológico, 49(4). Ministério da Saúde. http://portalarquivos2.saude.gov.br/images/pdf/2018/janeiro/31/2018-004-Hanseniase-publicacao.pdf

Brook, C. E., Beauclair, R., Ngwenya, O., Worden, L., Ndeffombah, M., Lietman, T. M., Satpathys, S. K., Galvani, A. P., \& Porco, T. C. (2015). Spatial heterogeneity in projected leprosy trends in India. Parasites \& Vectors (8).

Chaptini, C., Marshman, G. (2015). Leprosy: a review on elimination, reducing the disease burden and future research. Lepr. Rev., 86(4).

CNDSS. (2008). As causas sociais das iniquidades em saúde no Brasil. Relatório final da Comissão Nacional sobre Determinantes Sociais da Saúde. http://bvsms.saude.gov.br/bvs/publicacoes/causas_sociais_iniquidades.pdf

CSDH. (2008). Closing the gap in a generation: health equity through action on the social determinants of health. Final Report of the Commission on Social Determinants of Health. Geneva, World Health Organization.

Cunha, M. D., Almeida, A. S., Cunha, G. M., Santos, R. S. (2016). Geographic weighted regression: applicability to epidemiological studies of leprosy. Rev. Soc. Bras. Med. Trop., 49(1). 
Research, Society and Development, v. 10, n. 9, e16110917823, 2021

(CC BY 4.0) | ISSN 2525-3409 | DOI: http://dx.doi.org/10.33448/rsd-v10i9.17823

Freitas, L. R. S., Duarte, E. C., Garcia, L. P. (2017). Análise da situação epidemiológica da hanseníase em uma área endêmica no Brasil: distribuição espacial dos períodos 2001 - 2003 e 2010 - 2012. Rev bras epidemiol, 20(4).

Freitas, L. R. S., Duarte, E. C., Garcia, L. P. (2014). Leprosy in Brazil and its association with characteristics of municipalities: ecological study, 2009-2011 Trop Med Int Health, 19(10).

IBGE. Instituto Brasileiro de Geografia e Estatística. (2017) Censo demográfico 2010: resultados do universo por setor censitário.

Jannuzzi, P. M. (2012) Indicadores sociais na formulação e avaliação de políticas públicas. Flacso, 2012.

Londrina. Londrina em dados 2017. http://www1.londrina.pr.gov.br/index.php?option=com_content\&view=article\&id=543\&Itemid=558\&limitstart=4 .

Lopes, V. A. S, Rangel, E. M. (2014) Hanseníase e vulnerabilidade social: uma análise do perfil socioeconômico de usuários em tratamento irregular. Saúde Debate, 38(103).

Marmot, M. (2017). The health gap: Doctors and the social determinants of health. Scandinavian Journal of Public Health (45).

Montgomery, D. C., Peck, E. A., Vining, G. G. (2013). Intoduction to linear regression analysis. (4a ed.), John Wiley \& Sons.

Mongenstern, H. (2008). Ecologic Studies. In: Rothman KJ, Greenland S, Lash TL, editors. Modern Epidemiology. (3a ed.), Lippincot Williams \& Wilkins.

Nery, J. D., Pereira, S. M., Rasella, D., Penna, M. L. F., Aquino, R., Rodrigues, L. C. et al. (2014). Effect of the Brazilian conditional cash transfer and primary health care programs on the new case detection rate of leprosy. Plos Negl Trop Dis, 8(11).

Organização Mundial da Saúde. Comissão de Determinantes Sociais em $\quad$ Saúde. Relatório Final. http://determinantes.saude.bvs.br/docs/Relatorio_Final_CDSS_OMS.pdf

Organização Mundial da Saúde. (2016). Estratégia global para Hanseníase 2016-2020. Aceleração rumo a um mundo sem Hanseníase. http://apps.who.int/iris/bitstream/handle/10665/208824/9789290225201-pt.pdf,jsessionid=725AAE77B5BA16C6B74396590EBE847B?sequence=17

Organização Mundial da Saúde. (2016). Global leprosy update: time for action, accountability and inclusion. Wkly Epidemiol Rec, 91(36).

Ramos, A.C. V., Yamamura, M., Arroyo, L. H., Popolin, M. P., Chiaravalloti, N. F., Palha, P. F. et al. (2017). Spatial clustering and local risk of leprosy in São Paulo, Brazil. PLoS Negl Trop Dis, 11(2).

Silva, C. L. M., Fonseca, S. C., Kawa, H., Palmer, D. O. Q. (2017). Spatial distribution of leprosy in Brazil: a literature review. Rev Soc Bras Med Trop, 50(4),

Santos, S. D., Penna, G. O., Costa, M. C. N., Natividade, M. S., Teixeira, M. G. (2016). Leprosy in children and adolescents under 15 years old in an urban centre in Brazil. Mem Inst Oswaldo Cruz, 111(6). 